\title{
Tetrabenazine improves levodopa-induced peak-dose dyskinesias in patients with Parkinson's disease
}

\author{
Livia Brusa, MD, PhDa \\ Antonio Orlacchio, MD, PhD ${ }^{\text {b,c }}$ \\ Alessandro Stefani, MD ${ }^{\mathrm{c}, \mathrm{d}}$ \\ Salvatore Galati, MDe \\ Mariangela Pierantozzi, MD, PhDc,d \\ Cesare lani, MDa \\ Nicola Biagio Mercuri, MDc,d
}

\author{
a Neurology Unit, Sant'Eugenio Hospital, Rome, Italy \\ b Neurogenetics Laboratory, CERC-IRCCS Santa \\ Lucia, Rome, Italy \\ c Tor Vergata University Hospital Foundation, \\ University of Rome Tor Vergata, Rome, Italy \\ d Experimental Neurology Laboratory, CERC-IRCCS \\ Santa Lucia, Rome, Italy \\ e Neurocenter of Southern Switzerland, Lugano, \\ Switzerland
}

Correspondence to: Alessandro Stefani

E-mail: stefani@uniroma2.it

\section{Summary}

Since levodopa-induced peak dyskinesias (LIDs) may reflect, in part, a disproportionate phasic release of dopamine from synaptic vesicles, we examined the ability of the vesicular depletor tetrabenazine (TBZ) to reduce LIDs in 10 dyskinetic advanced Parkinson's disease (PD) patients. After basal evaluation, the patients received, through a slow titration, oral TBZ twice a day for six weeks (up to $50 \mathrm{mg}$ daily) before being re-assessed after a challenge with levodopa. The primary outcome measure was the change in the Unified Parkinson's Disease Rating Scale (UPDRS) dyskinesia score (items 32 to 34 ).

TBZ was well tolerated. A clear treatment effect on LIDs emerged (up to $45 \%, p<0.05$ ). In two patients a little worsening of motor performance necessitated an increase of the antiparkinsonian therapy, which did not worsen peak-dose LIDs. The patients experienced a clear benefit in terms of their quality of life. In this open-label pilot study, orally administered TBZ resulted in objective and subjective improvements in LIDs. Larger pharmacological studies are in progress.

KEY WORDS: Parkinson's disease, peak dyskinesia, quality of life, vesicular dopamine, VMAT2
Introduction

There has long been debate over what strategies should be adopted in order to reduce the main complication of levodopa (LD) treatment of Parkinson's disease (PD), namely, levodopa-induced dyskinesias (LIDs) (Jenner, 2008). The dominant view is that it is necessary to obtain continuous activation of dopamine (DA) receptors (Stocchi et al., 2005), in other words, to attenuate the excessive and pulsatile stimulation of these receptors which results in hyperkinetic movements. The current approaches, aside from deep brain stimulation, which allows a reduction in the use of the dopaminominetics, include fine titration of LD intake, the addition of amantadine as the prototypic open-channel blocker acting at NMDA receptors, and the use of direct, long-acting DA receptor agonists. In addition, an appreciable control of involuntary movements has been obtained by dopaminergic stimulation, through either constant subcutaneous injections of apomorphine or continuous administration of LD methyl ester (Jenner, 2008; Stocchi et al., 2005). All these approaches still deserve attention (management of the condition in daily clinical practice actually involves a combination of them), although their results can sometimes prove disappointing in the long-run, especially in very advanced patients afflicted by co-morbidities (Stefani et al., 2010). Not surprisingly, there has emerged a large body of investigations addressing possible alternative approaches. These include the use of: agents modulating preynaptic release of glutamate, NMDA receptor NR2B subunit antagonists, adenosine A2A antagonists such as istradefylline, serotonin 5$\mathrm{HT}(1 \mathrm{~A})$ and $5-\mathrm{HT}(1 \mathrm{~B})$ agonists, phosphodiesterase inhibitors, molecules affecting the endocannabinoid system, and even atypical neuroleptics (Meco et al., 2009); for representative reviews on this topic see Jenner (2008) and Stefani et al. (2010). However, despite success in primate models and promising results in clinical trials, the overall scenario remains disappointing, with different regulatory agencies failing to find sufficient evidence to allow the transfer of these preliminary data into clinical practice (Horstink et al., 2006; Pahwa et al., 2006; Hayden et al., 2009).

Here, we propose a radically different approach, which is based on the dynamics of DA formation and release from nerve terminals and involves a challenge with LD. This approach takes into account the different mechanisms of DA release: a) action potentialdependent release (phasic) from vesicles, and b) metabolically-regulated release (tonic), which is largely independent of the vesicular stores of DA. 
Our basic hypothesis is that agents capable of interfering with the phasic vesicular DA reservoir might prove to be beneficial. In this context, tetrabenazine (TBZ) is known to effectively deplete DA storage. For instance, as shown through fast scan cyclic voltammetry in freely-moving rats, TBZ almost abolished phasic DA release in the accumbens "and also increased extracellular dopamine by inducing reverse transport by dopamine transporter" (Owesson-White et al., 2012). In the US, TBZ has been recommended as the first-choice drug for the treatment of hyperkinetic disorders (Armstrong and Miyasaki, 2012).

Hence, in this study we set out to verify whether TBZ might, by inhibiting the phasic whilst maintaining the tonic LD-induced DA release in the brain, prove to be therapeutically useful in the control of LIDs in PD patients.

To explore this hypothesis, we examined the effects of TBZ in dyskinetic PD patients, and found that it clearly reduced the severity of LIDs.

\section{Materials and methods}

Ten advanced idiopathic PD patients suffering from disabling peak-dose dyskinesias following LD ingestion were enrolled (Table 1). Idiopathic PD was diagnosed according to the Brain Bank criteria. Antiparkinsonian medications producing the best control of PD and LID symptoms were administered at fixed doses for at least one month prior to and during the study. Inclusion criteria were: stable medication dose for four weeks, and LIDs $>25 \%$ of waking hours (corresponding to a score of $\geq 2$ on item 32 of the Unified Parkinson's Disease Rating Scale, UPDRS) that are bothersome to the patient (a score of $\geq 2$ on item 33 ). It is to be noted that our group was small but quite homogeneous in terms of disease duration, early initiation of LD-based therapy, and relatively high LDequivalent daily dose. Informed consent was obtained from all the individuals participating in the study, which was performed according to a protocol reviewed and approved by the ethics committee of the Istituto di Ricovero e Cura a Carattere Scientifico Santa Lucia.
Patients initially underwent a basal evaluation. This first session took place in the morning, following an overnight fast, and after the patients had been administered immediate-release LD-carbidopa in a dose corresponding to $125 \%$ of their usual morning LD equivalent dose. The evaluation session was video-recorded and the UPDRS-III (all parts of the scale) and Abnormal Involuntary Movement Scale (AIMS) were used for scoring. The AIMS scores were calculated as the sum of the individual scores for facial/oral movements (0-16), extremity movements (0-8) and trunk movements (0-4). Each item within these categories was scored as 0 , none; 1 , mild; 2, moderate; 3 , severe; and 4, extreme. The UPDRS-III and AIMS scores were calculated immediately after LD-carbidopa administration, and every 15 minutes thereafter for one hour (t0, t15, t30, t45, t60). Two blinded raters, both experts in the field of movement disorders, rated videotapes independently to provide AIMS and UPDRS scores.

Following the first evaluation, TBZ was administered twice a day with a slow titration consisting of the introduction of $6.25 \mathrm{mg}$ per week, up to $12.5 \mathrm{mg}$ bid. Each patient was evaluated during a second session, six weeks after the beginning of the drug treatment (hence, after $>4$ weeks of steady-state regimen); the evaluation setting was exactly the same as used for the first assessment. If a patient showed a worsening of the UPDRS-III score, $30 \%$ of the total LD daily dose was added, and a third evaluation was performed a further four weeks later. Through comparison of weekly diaries (one kept during the week before starting TBZ and one kept during the last week under TBZ treatment), it was also possible to gather insights into the subjective impact of this agent on the subject's own perception of daily performance.

\section{Statistical analysis}

The Wilcoxon test was performed to compare the various AIMS and UPDRS values (t15; t30; t45; t60) between the two assessments, i.e. before and after the six weeks of TBZ treatment (using a Bonferroni corrected $p$-value of $<0.012$ ).

For all statistical analyses, a p-value of $<0.05$ was considered to be significant.

Table 1 - Demographic and clinical features of the 10 patients.

\begin{tabular}{lc}
\hline Age (years, mean \pm SD) & $71.7 \pm 7.55$ \\
Females/males & $4 / 6$ \\
Disease duration (years, mean \pm SD) & $13.6 \pm 3.11$ \\
Hoehn \& Yahr scale score (mean \pm SD) & $2.75 \pm 0.26$ \\
Duration of L-dopa treatment (years, mean \pm SD) & $10.1 \pm 3.09$ \\
LEDD (mg, mean \pm SD) & $1080 \pm 250$ \\
UPDRS On/Off (mean \pm SD) & $24.8 \pm 7.0 / 41.2 \pm 7.7$ \\
PDQ-39 (summary index, mean \pm SD) & $42.4 \pm 12$ \\
\hline
\end{tabular}

Abbreviations: LEDD=Levodopa-equivalent daily dose; UPDRS=Unified Parkinson's Disease Rating Scale; $\mathrm{PDQ}-39=$ Parkinson's Disease Questionnaire-39. 


\section{Results}

Following the TBZ treatment, the dyskinesia score obtained using the AIMS was found to be significantly decreased in comparison with that recorded at the baseline evaluation. This difference was evident at $\mathrm{t} 15$ (Wilcoxon test $Z=-2.41 ; \mathrm{p}=0.011)$, t30 ( $Z=-2.52$; $\mathrm{p}=0.012), \mathrm{t} 45(Z=-2.53 ; \mathrm{p}=0.011)$ and $\mathrm{t} 60(Z=-2.55$; $p=0.011)$. The UPDRS-III performance of the whole group was not significantly modified after TBZ treatment at $\mathrm{t} 15(Z=-2.05 ; \mathrm{p}=0.055)$, $\mathrm{t30}(Z=-2.03$; $\mathrm{p}=0.042)$ or $\mathrm{t} 60(Z=-1.70 ; \mathrm{p}=0.089)$ (Fig. 1). However, two patients experienced a slight worsening of motor performance and, in accordance with the study design, were submitted to an increase of the daily LD dose $(+30 \%)$ and a further clinical evaluation in the same setting as used for the first and the second evaluations (administering 125\% of their usual morning LD dose). This last evaluation revealed an improvement in the UPDRS score compared to the baseline evaluation, while the improved dyskinesia score persisted (data not shown).

A subjective evaluation of motor performance (based on the daily diaries kept by the patients) revealed a beneficial effect of TBZ on the global incidence of disabling dyskinesia (considered on a weekly basis, this was found to be reduced from $20 \%$ to $9 \%$, Fig. 2); this improvement was not associated with a significant worsening of the global "on" phase (from $47 \%$ to $46 \%$, Fig. 2).

No serious adverse events or significant abnormal laboratory test results were noted. In addition, the patients did not show any substantial difference

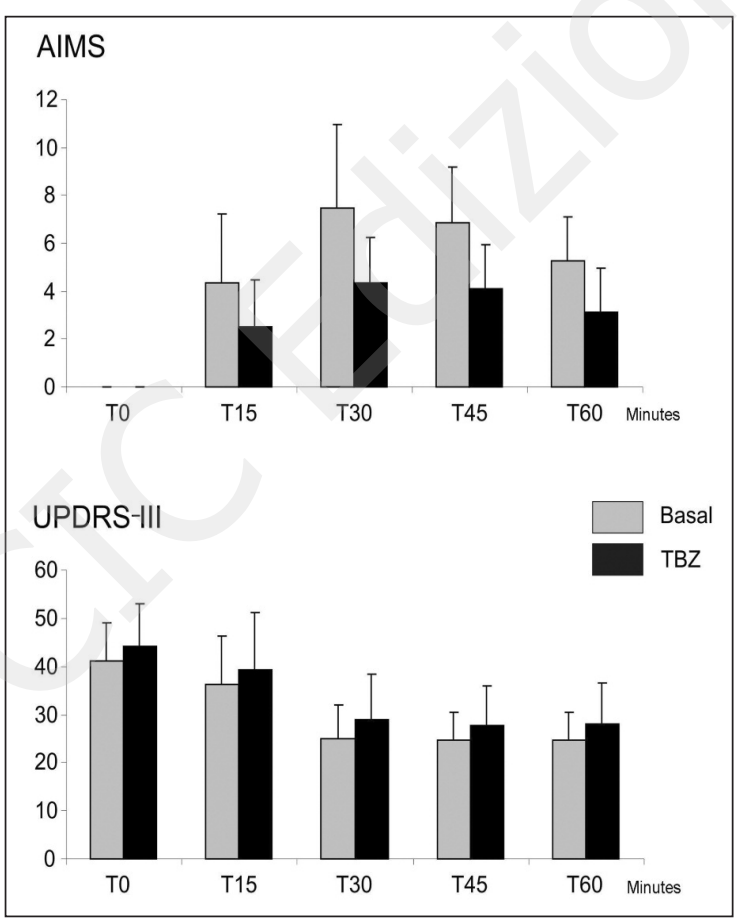

Figure 1 - Evaluation of the effects of tetrabenazine on AIMS and UPDRS scores. Note the ameliorating effect of TBZ on the AIMS values ( $p<0.05$ for all time points).

\section{Patient's diary}

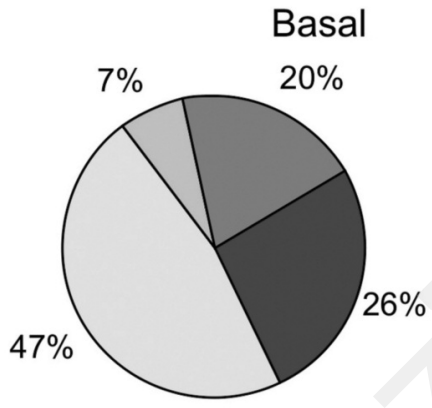

TBZ

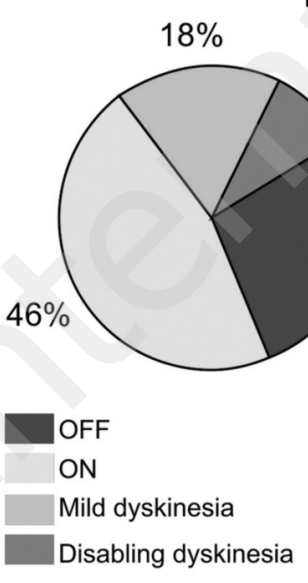

Figure 2 - Percentage of "off" time, "on" time, and "on" time with mild and disabling dyskinesia at baseline and under TBZ treatment. Note the reduction of the disabling dyskinesia during TBZ treatment without a significant worsening of the global "on" phase.

between Hospital Anxiety and Depression Scale scores recorded before TBZ treatment and at the end of the study.

\section{Discussion}

Long-term dopaminergic therapy for PD commonly results in motor complications including "on-off" fluctuations and dyskinesias, but it is still unclear how best to assess treatment effects on dyskinesias in clinical trials (Olanow et al., 2009). The precise impact of LIDs on the quality of life of severe PD patients may vary depending on patient's age, co-morbidities and treatment history. Decades ago, when we were not even aware of the possibility of pursuing the option of "continuous dopaminergic stimulation" (CDS) in order to replicate the pattern of endogenous tonic DA release, patients were more likely to experience early and severe LIDs. Today's neurologists, on the other hand, are more culturally aware of CDS-based therapeutic strategies and have often been trained in their use. This, together with a greater understanding of LID 
pathogenesis (Jenner, 2008; Calabresi et al., 2010), is reducing the risk of early initiation of highly pulsatile drug regimens, and thus minimizing and postponing the onset of LIDs. However, given the involvement of mechanisms intrinsic to the disease progression, LIDs are, to a large extent, independent of the treatment per se (Napolitano et al., 2010). In fact, initial LD administrations can trigger LIDs in previously LD-naïve subjects. This phenomenon is largely dependent upon an early derangement of the presynaptic machinery. Dyskinetic patients indeed suffer from an increased DA turnover (Lunardi et al., 2009) and altered DA transporter expression (Troiano et al., 2009). PET studies, for instance, have shown that "the relationship between putaminal methylphenidate/dihydrotetrabenazine ratio and the presence of dyskinesia was not altered after correcting for age, treatment, and measures of disease severity" (Troiano et al., 2009).

The present work addressed the possibility that treatment with low-dose TBZ, used for decades to treat a variety of hyperkinetic movement disorders including tardive dyskinesia (Martí-Massó and Obeso, 1985; Jankovic and Orman, 1988), may reduce the intensity of peak-dose LIDs in a small cohort of PD patients. TBZ causes depletion of monoamines from nerve terminals due to its ability to reversibly inhibit the vesicular monoamine transporter 2 (VMAT2), hence impairing DA translocation into synaptic vesicles for storage and subsequent release (Zheng et al., 2006).

Experimental data have shown that: a) depletion of the neuronal DA content using TBZ or reserpine produces a parkinsonian syndrome that is reversed by LD (Giladi and Melamed, 1999; Mercuri et al.,1998), and b) the phasic release of DA subsequent to synaptic activation is reduced by its vesicular depletion while the conversion of LD into DA, which modulates tonic release, is partially independent of the conservation of the intracellular pool (Xu and Dluzen, 1998; personal unpublished observation). With these premises in mind, we administered TBZ to 10 PD patients with disabling peak-dose LIDs. The most significant result obtained was actually a remarkable change in the quality of LIDs, which became tolerable for most of the day without de-titration of the usual LD dose.

The present open-label study showed a reduction in dyskinesia scores in TBZ-treated PD patients after a challenge with LD. Only a minority of patients $(n=2)$ showed a small increase in the UPDRS score, but this could be corrected (without concomitant worsening of LIDs) by increasing the dose of LD. Therefore, it appears that, by reducing the phasic release of DA, consequently avoiding pulsatile receptor stimulation and facilitating tonic dopaminergic stimulation, TBZ ameliorates peak-dose LIDs.

The functional benefit of reducing dyskinesias was not associated with serious adverse effects. Our observation seems to exclude a large influence of TBZ on psychic domains. However, since the treatment with TBZ lasted only six weeks, longitudinal studies are needed to assess its long-term benefits or risks.

To date, the long-term tolerability of TBZ has been val- idated only in hyperkinetic disorders without parkinsonian signs. TBZ is in fact the only drug approved for Huntington's disease by a regulatory agency, and only for the treatment of chorea (Armstrong and Miyasaki, 2012; Mestre and Ferreira, 2012). However, ongoing clinical practice may show it to offer potential benefits in a broader spectrum of diseases.

\section{Acknowledgments}

This study was supported by the Italian Ministry of Health (grants no.s EBRI1.O, PS05.11, PS05.21, and REG.17O to A.O.; grants no.s PS05.15M, RF.06.70M, and RF07.96M to N.B.M.), as well as the Comitato Telethon Fondazione Onlus, the Amministrazione Autonoma dei Monopoli di Stato (AAMS), and the Italian city of Gubbio (grant no. GGP06209 to A.O.).

\section{References}

Armstrong MJ, Miyasaki JM, et al (2012) Evidence-based guideline: pharmacologic treatment of chorea in Huntington disease: report of the guideline development subcommittee of the American Academy of Neurology. Neurology 79:597603.

Calabresi P, Di Filippo M, Ghiglieri V, et al (2010). Levodopa-induced dyskinesias in patients with Parkinson's disease: filling the bench-to-bedside gap. Lancet Neurol 9:1106-1117.

Giladi N, Melamed E (1999). Levodopa therapy can ameliorate tetrabenazine-induced parkinsonism. Mov Disord; 14:158159.

Hayden MR, Leavitt BR, Yasothan U, et al (2009). Tetrabenazine. Nat Rev Drug Discov 8:17-18.

Horstink M, Tolosa E, Bonuccelli U, et al (2006) Review of the therapeutic management of Parkinson's disease. Report of a joint task force of the European Federation of Neurological Societies (EFNS) and the Movement Disorder Society-European Section (MDS-ES). Part II: late (complicated) Parkinson's disease. Eur J Neurol 13:1186-1202.

Jankovic J, Orman J (1988). Tetrabenazine therapy of dystonia, chorea, tics, and other dyskinesias. Neurology 38:391-394.

Jenner P (2008). Molecular mechanisms of L-DOPA-induced dyskinesia. Nat Rev Neurosci 9:665-677.

Lunardi G, Galati S, Tropepi D, et al (2009). Correlation between changes in CSF dopamine turnover and development of dyskinesia in Parkinson's disease. Parkinsonism Relat Disord 15: 383-389.

Martí-Massó JF, Obeso JA (1985). Coprolalia associated with hemiballismus: response to tetrabenazine. Clin Neuropharmacol 8:189-190.

Meco G, Stirpe P, Edito F, et al (2009). Aripiprazole in L-dopainduced dyskinesias: a one-year open-label pilot study. J Neural Transm116: 881-884.

Mercuri NB, Scarponi M, Federici M, et al (1998). Modification of levodopa responses by deprenyl (selegiline): an electrophysiological and behavioral study in the rat relevant to Parkinson's disease. Ann Neurol 43:613-617.

Mestre TA, Ferreira JJ (2012). An evidence-based approach in the treatment of Huntington's disease. Parkinsonism Relat Disord 18:316-320.

Napolitano F, Bonito-Oliva A, Federici M, et al (2010). Role of aberrant striatal dopamine D1 receptor/cAMP/protein kinase A/DARPP32 signaling in the paradoxical calming effect of amphetamine. J Neurosci 30: 11043-11056 
Olanow CW, Stern MB, Sethi K (2009). The scientific and clinical basis for the treatment of Parkinson disease. Neurology 72:S1-S136.

Owesson-White CA, Roitman MF, Sombers LA, et al (2012). Sources contributing to the average extracellular concentration of dopamine in the nucleus accumbens. J Neurochem 121:252-262

Pahwa R, Factor SA, Lyons KE, et al (2006). Practice Parameter: treatment of Parkinson disease with motor fluctuations and dyskinesia (an evidence-based review): report of the Quality Standards Subcommittee of the American Academy of Neurology. Neurology; 66:983-995.

Stefani A, Pierantozzi M, Koch G, et al (2010). Therapy for dyskinesias in Parkinson's disease patients. Future Neurology
5:277-299.

Stocchi F, Vacca L, Ruggieri S, et al (2005). Intermittent vs continuous levodopa administration in patients with advanced Parkinson disease: a clinical and pharmacokinetic study. Arch Neurol 62:905-910.

Troiano AR, de la Fuente-Fernadez R, Sossi V, et al (2009) PET demonstrates reduced dopamine transporter expression in PD with dyskinesias. Neurology 72: 1211-1216.

Xu K, Dluzen DE (1998). Alteration in L-DOPA evoked dopamine and DOPAC output under conditions of impaired vesicular dopamine storage. J Neural Transm 105:1091-1101.

Zheng G, Dwoskin LP, Crooks PA (2006). Vesicular monoamine transporter 2: role as a novel target for drug development. AAPS J 8:E682-E692. 